\title{
Pituitary luteinizing hormone responses to single doses of exogenous GnRH in female social Cape ground squirrels exhibiting low reproductive skew
}

\author{
- $\quad$ T. P. Jackson ${ }^{1}$, \\ - J. M. Waterman ${ }^{2} \&$ \\ - $\quad$ N. C. Bennett ${ }^{3}$
}

1 Conservation Ecology Research Unit, Department of Zoology and Entomology, University of Pretoria, Pretoria, South Africa

2 Department of Biology, University of Central Florida, Orlando, FL, USA

3 Mammal Research Institute, Department of Zoology and Entomology, University of Pretoria, Hatfield, South Africa

\section{Correspondence}

T. P. Jackson, Conservation Ecology Research Unit, Department of Zoology and Entomology, University of Pretoria, Pretoria, South Africa. Email: tjackson@zoology.up.ac.za

[Figures at the bottom of the document]

\begin{abstract}
The Cape ground squirrel Xerus inauris is unusual among social mammals as it exhibits a low reproductive skew, being a facultative plural breeder with not all females breeding within a group. We investigated pituitary function to assess whether there was reproductive inhibition at the level of the pituitary and potentially the hypothalamus in breeding and non-breeding female Cape ground squirrels. We did so during the summer and winter periods by measuring luteinizing hormone (LH) responses to single doses of $2 \mathrm{~g}$ exogenous gonadotropin-releasing hormone $(\mathrm{GnRH})$ and physiological saline
\end{abstract}


administered to 42 females from 11 colonies. Basal LH concentrations of females increased in response to the $\mathrm{GnRH}$ challenge. Basal plasma $\mathrm{LH}$ concentrations were greater during winter, when most oestrus events are observed. However, we found no differences in plasma LH concentrations between breeding and non-breeding females. We showed that the anterior pituitary of non-breeding female ground squirrels is no less sensitive to exogenously administered GnRH than that of breeding females. We therefore concluded that the pituitary is no more active in breeding than non-breeding females. The lack of differentiation in response to GnRH suggests that either non-breeding females have ovaries that are less sensitive to LH or that they refrain from sexual activity with males through an alternative mechanism of self-restraint.

\section{Introduction}

Of the 63 species of mammal (excluding primates) recorded living in multi-generational family groups, $90 \%$ breed cooperatively (Emlen, 1997). In cooperative species, nonbreeding individuals help raise the young of dominant breeders (Clutton-Brock, 2002). Central to our understanding of cooperation is the degree to which socially dominant individuals monopolize breeding opportunities within a group. To date, the majority of cooperatively breeding mammals studied exhibit a high degree of reproductive skew, with little or no reproductive opportunities afforded to socially subordinate group members (Clutton-Brock, 2002). For example, the proportion of breeding attempts in which more than one female is pregnant is only $20 \%$ for meerkats Suricata suricatta (Clutton-Brock et al., 1999) and 27\% for dwarf mongooses Helogale parvula (Creel \& Waser, 1991, 1997). In alpine marmots Marmota marmota and golden marmots, Marmota caudate aurea, only one female breeds in a social group (Blumstein \& Armitage, 1999). Hence, in these species a single pair of animals is involved in reproduction and few breeding opportunities exist for subordinate individuals. Reproductive skew occurs due to an unequal distribution of reproduction among members of a social group. Dominant control can maintain reproductive skew (Vehrencamp, 1983), in which case the socially dominant individual(s) maintain reproductive suppression of the non-breeding, socially subordinate group members (Faulkes \& Bennett, 2001). Several mechanisms may mediate reproductive suppression. 
Behavioural mechanisms include infanticide and the behavioural exclusion of subordinates from mating opportunities. Alternatively, changes in the physiology of nonbreeders may lead to an inhibition of reproduction (such as puberty delay), suppression of ovulation, blocks to implantation and spontaneous abortion (Snowdon, 1996; Wingfield \& Sapolsky, 2003). Physiological suppression may be mediated via the hypothalamicpituitary-adrenal axis, through the release of corticosteroids into the circulatory system (Wingfield \& Sapolsky, 2003). On the other hand, non-breeders may occur in groups through self-restraint, whereby maturity is delayed and females do not attempt reproduction until an unrelated male is available for mating (Snowdon, 1996). Incidences of low reproductive skew (i.e. plural breeding) among cooperative breeding mammals are rare. Examples include yellow-bellied marmots Marmota flaviventris (Armitage \& Schwartz, 2000), banded mongooses Mungos mungo (Cant, 2000) and the Cape ground squirrel Xerus inauris (Waterman, 2002). Banded mongooses are unusual in that breeding is highly synchronous between females and more than five females may come into oestrus within a week (Cant, 2000). Breeding is also fairly synchronous in yellow-bellied marmots, as mating follows the emergence of females from hibernation (Schwartz \& Armitage, 2005). In Cape ground squirrels, oestrous appears to be less synchronous, both between and among social groups, while females can breed at any time of year (Waterman, 1996, 1998). Despite plural breeding, Waterman (2002) suggested that a delay in female maturity might suppress reproduction for these squirrels. The age of first breeding is also group-size dependent, with females in smaller groups reaching sexual maturity at an earlier age than those in groups with more breeding females (Waterman, 2002).

Typical of many sciurids, philopatry characterizes female Cape ground squirrel social groups. A single social group, which usually comprises two to three breeding females and up to nine non-breeders of either sex, share sleeping burrows and a common feeding range (Waterman, 1995). Burrow clusters are usually separated from each other by more than $100 \mathrm{~m}$ (Waterman, 1995). Gestation lasts for a period of 48 days, after which females give birth to one or two young, which are weaned 52 days later (Waterman, 1996). Aggressive interactions are extremely rare in such groups (Waterman, 1995; Pettitt, 2006), although the age asymmetry between breeding females and their young 
may assure a dominance asymmetry (Emlen, 1996). Given the social structure of female Cape ground squirrel, where not all females reproduce, we suggest that physiologically mediated delays in maturity (Batzli, Getz \& Hurley, 1977; Massey \& Vandenbergh, 1980; Wasser \& Barash, 1983; Coppola \& Vandenbergh, 1985; Kruczek \& Marchlewska-Koj, 1986) may underlie reproductive suppression. By challenging the pituitary with an excess dose of exogenous GnRH, we can infer whether the pituitary of breeding versus non breeding females varies in response and hence infer whether the two groups differ in the synthesis and subsequent release of this gonadotrophin. This experimental approach allows us to investigate the hypothalamicpituitary-gonadal axis in a relatively non-invasive way. Physiological suppression at the level of the pituitary would be interpreted if the response of one group was reduced to that of the other as has been found in socially suppressed mole-rats (Bennett, Faulkes \& Molteno, 1996; Bennett, Faulkes \& Spinks, 1997). In physiologically suppressed social species, inhibition of reproduction usually results from a centrally mediated action of corticotrophic-releasing hormone and $\beta$-endorphin on the $\mathrm{GnRH}$ release and direct action of glucocorticoids at the level of the hypothalamic-pitutiary-gonadal axis. However, a lack of difference in a challenge would suggest that the animals are not physiologically suppressed in the pituitary region through the hypothalamic-pituitary-adrenal axis but rather the ovaries could be less sensitive to luteinizing hormone (LH) or the females may be avoiding breeding due to other factors such as incest avoidance (Bennett et al., 1997). Accordingly, we made the following a priori predictions: breeding and non-breeding adult female Cape ground squirrels would show (1) differences in circulating concentrations of baseline LH; (2) there would be a status-related difference in the pituitary responsiveness to exogenous $\mathrm{GnRH}$; and (3) a seasonal difference occurs in the response.

\section{Materials and Methods}

\section{Study site}

We conducted our study at the SA Lombard Nature Reserve, near Bloemhof in the NorthWest Province, South Africa $\left(25^{\circ} 29^{\prime} \mathrm{E}, 27^{\circ} 38^{\prime} \mathrm{S}\right)$. The reserve receives an annual rainfall of $500 \mathrm{~mm}$, falling predominantly from November to April through the austral summer 
(Herzig-Straschil, 1978). We located ground squirrel colonies in an area of open grassland on the reserve. Fieldwork was conducted during November 2002 and late May 2003. We categorized these as summer and winter trapping periods, respectively.

\section{Animal handling}

We used Tomahawk Live Traps (Tomahawk Live Trap Co., Tomahawk, WI, USA) baited with a mixture of peanut butter and grain to capture ground squirrels. We handled these animals within an hour of capture and used a cloth handling-cone (Koprowski, 2002) to restrain individuals. Animals could be held in this manner to weigh them and then for the 20 min duration of the physiological trial. All research protocols were approved by the animal care committees at the Universities of Central Florida and Pretoria, and complied with their Guidelines for Animal Research.

In total, we sampled 42 individuals captured from 11 colonies. For one individual, reproductive status changed between periods and we used data from this individual as both a breeder and non-breeder. We also used data from seven individuals that were caught twice to examine the effects of a saline placebo on circulating LH, independently of our analyses of reproductive status and season. The ground squirrels at the study site had been monitored for 1 year and were individually marked.

\section{Assessment of reproductive status}

We distinguished breeding from non-breeding females using a number of criteria. Breeding females (reproductively mature individuals that had experienced at least one oestrus) had larger vulvas and prominent nipples ( $\sim 1 \mathrm{~cm}$ long) than non-breeders. The nipples of breeding females swell during the first gestation and remain permanently swollen thereafter (Waterman, 1995). During lactation, these nipples increase to almost $2 \mathrm{~cm}$ in length (Waterman, 1996). Non-breeding females were characterized by their rudimentary nipples ( $<0.5 \mathrm{~cm}$ long) and small vulvas. In addition, we were able to document the behaviour associated with the oestrus cycles of breeding females (including first oestrus) and to determine the times when females were gestating and lactating. 


\section{GnRH and physiological saline administration}

We took a pre (basal) blood sample to measure basal circulating LH concentrations and subsequently challenged the pituitary with a $2 \mu \mathrm{g}$ exogenous $\mathrm{GnRH}$ challenge to release all potential stored LH and obtained a post (response) LH concentration. This is an acceptable method that has been used in a number of studies (see Abbott, 1984 for marmosets; Faulkes et al., 1990; Faulkes, Abbott \& Jarvis, 1991 for naked mole-rats Heterocephalus glaber; Bennett et al., 1993, 1996 for Damaraland mole-rats Cryptomys damarensis; and O'Riain et al., 2000 for meerkats). We stored ampoules of $2 \mu \mathrm{g}$ of GnRH in $200 \mu \mathrm{L}$ of sterile physiological saline or sterile $200 \mu \mathrm{L}$ physiological saline only (control) at $-20^{\circ} \mathrm{C}$ until required. The GnRH was synthesized in the laboratory of R. P. Millar, using solid-phase methodology and the purity of GnRH was $>98 \%$ homogeneity (Millar et al., 1989). We collected blood samples (300-400 $\mu \mathrm{L}$ whole blood) directly from the femoral vein by capillary action using heparinized micro-haematocrit tubes and a 21-gauge needle. This femoral vein could easily be identified once the surrounding fur had been shaved. We took an initial blood sample to measure the circulating tonic LH concentration in the blood. Following this, we administered a dose of GnRH or physiological saline to the squirrel. We administered doses sub-cutaneously as a bolus $200 \mu \mathrm{L}$ injection. Blood samples were taken from the squirrels immediately before and 20 min after this injection. Following collection, the samples were chilled before centrifuging at 2000 g, using a Labnet 'Spectrafuge Mini' centrifuge (Labnet International Inc., Woodbridge, NJ, USA) and plasma stored at $-20^{\circ} \mathrm{C}$.

\section{LH bioassay}

We measured LH concentrations in an in vitro bioassay based on the production of testosterone by mouse Leydig cells (van Damme, Robertson \& Diczfalusy, 1974). Details of the assay are described in Harlow et al. (1984) and Hodges et al. (1987). We used a bioassay in preference to a standard RIA because the bioassay provides a measure of biologically active LH. The LH in the sample acts on the Leydig cells of the mouse preparation, resulting in the production of testosterone. Hence, the bioassay provides us with a measure of LH that is biologically active. 
We assayed plasma samples at a dilution of 1:20. LH pituitary preparation (2nd International Standard 1988, code no. 80/552, NIBSC, UK) over the range 200-1.4 miu $\mathrm{ml}^{-1}$. The testosterone produced by the Leydig cells was measured by radioimmunoassay as described by Bennett (1994). Checks for parallelism to the standard curve were carried out to validate the LH assay after GnRH administration. Following logit-log transformation of the data (Chard, 1987), parallelism of the LH standard and the serial dilution of squirrel plasma were tested using Statistica software (Statsoft, Tulsa, OK, USA). The curve was parallel and not significantly different from the reference preparation (ANCOVA $F=2.14$, d.f. $=1,6, P=0.194$ ). The sensitivity of the assay (determined at $90 \%$ binding) was $12.7 \mathrm{mIU}^{-1} \mathrm{bee}^{-1}$ or $2.5 \mathrm{miu} \mathrm{ml}^{-1}$. We modelled standard curves using the curve fit option of Sigmaplot. Intra and inter-assay coefficients of variation for repeated measurement of a quality control were $8 \%(n=4)$ and $11 \%(n=8)$, respectively.

We used a twoway ANOVA to examine differences in the body mass of females in relation to status (breeding, non-breeding) and season (summer, winter). As the hormonal data were not normally distributed, we used non-parametric Wilcoxon matched pairs statistics (Zar, 1996) to examine the effects of treatment (pre- and post-injection of GnRH or saline) on circulating LH concentrations. In order to examine the effects of breeding status (breeding, non-breeding) and time of year (summer, winter) on circulating LH concentrations, we used a twoway repeated measures ANOVA design with breeding status (breeding, non-breeding) and time of year (summer, winter) as the categorical factors. We treated the effect of treatment on the same individual (pre- and post injection of GnRH or saline) as the repeated measure. To conduct this analysis, we first applied a reciprocal transformation on our data (see Zar, 1996), in order to meet the assumptions of a parametric design. All statistical tests are two-tailed and we assume a significant result for $P<0.05$. The results of body mass and LH concentrations are provided in the results as mean values \pm SE. 


\section{Results}

\section{Female body mass}

There was a significant effect of status, but not season on female body mass. The body mass of breeding females $635 \pm 14 \mathrm{~g}(n=28)$ and that of non-breeding females $585 \pm 20 \mathrm{~g}$ $(n=14)\left(F_{1,38}=4.11, P<0.05\right)$, while during summer females weighed $596 \pm 16 \mathrm{~g}$, compared with $624 \pm 18 \mathrm{~g}$ through winter $\left(F_{1,38}=1.33, P=0.26\right)$.

\section{Saline versus GnRH challenge}

Comparing circulating plasma LH concentrations between the saline placebo and GnRH females before treatment, these did not differ significantly $\left(U_{17,10}=64, P=0.29\right.$; Fig. 1). Circulating LH levels of individuals did not increase significantly in response to the placebo challenge of physiological saline, but did increase in response to an injection of GnRH (saline: $t=22, n=10, P=0.57$; GnRH challenge: $t=28, n=17, P=0.02$; Fig. 1 ).

\section{Social status, season and GnRH challenge}

Our results suggest that there was no effect of reproductive status on the concentrations of $\mathrm{LH}\left(F_{1,27}=1.54, P=0.22\right.$; Fig. 2). LH concentrations were, however, higher during winter than summer $\left(F_{1,27}=6.17, P=0.019\right.$; Fig. 3$)$, while there was no interaction between reproductive status and season $\left(F_{1,27}=0.49, P=0.49\right)$. The most significant increase we recorded was an increase in concentrations of LH concentrations, post-GnRH challenge of individuals $\left(F_{1,27}=9.63, P=0.004\right)$.

\section{Discussion}

Our study demonstrates that female Cape ground squirrels respond to a GnRH challenge, with the pituitary releasing the stored LH from the gonadotrophin cells. Interestingly, breeders did not exhibit enhanced circulating basal concentrations of LH when compared with non-breeders and likewise the magnitude of the response was not dependent on breeding status. Typically, while breeding may be asynchronous through the year, a higher proportion of oestrus events have been recorded in this species during winter 
(Herzig-Straschil, 1978), a time when we recorded higher basal concentrations of plasma LH.

Female Cape ground squirrels reach sexual maturity from 8 to 10 months (Waterman, 1996, 2002), when they are expected to attain a mass of 540-580 g. Our study, however, suggests that the mean mass of non-breeding is around $585 \mathrm{~g}$ and we therefore conclude that many of the non-breeding females in our study, while old enough to reproduce, did not do so. From these observations, it is easy to conclude that these non-breeders are reproductively repressed by either behavioural or physiological means (see Waterman, 2002). The dominant control model of reproductive skew posits that reproductive suppression of the non-breeding, socially subordinate group members is maintained by the socially dominant individual(s). Control may either be through behavioural or physiological mechanisms. The social system of female groups of Cape ground squirrels is at odds with the dominant control model. Firstly, these squirrels are unusual, as aggression is usually very low between females (Waterman, 1995, 2002), and no dominance hierarchy exists among breeding females (Waterman, 1995). We therefore argue that behaviourally mediated domination via aggression of individuals does not exist within female squirrels.

In mammals from several different taxa, physiological suppression can be measured at the level of the pituitary. Physiological suppression is usually manifested as a reduced pituitary secretion of LH or decreased sensitivity to hypothalamic GnRH (Faulkes et al., 1990; Bennett et al., 1993; O'Riain et al., 2000; Wingfield \& Sapolsky, 2003). We did not observe differences in the basal concentrations of bioactive LH or pituitary responses to an exogenous GnRH challenge between breeders and non-breeders. We contend that in Cape ground squirrels, physiological suppression does not operate to the degree illustrated in some other social cooperatively breeding mammals including Damaraland mole-rats C. damarensis and naked mole-rats H. glaber (Faulkes et al., 1990; Bennett et al., 1993).

Instead, we suggest that in female Cape ground squirrels reproductive inhibition does not involve physiological suppression at the pituitary level. We also argue that it is unlikely that suppression occurs at the hypothalamic level either, as the pituitary is responding to a GnRH challenge similarly in both breeding and non-breeding ground squirrels. This 
indicates that there is no downregulation of GnRH receptors on the pituitary and thus GnRH is probably released normally in female squirrels of either status. This merits further investigation where circulating cortisol concentrations may provide insights as to the levels of stress that are experienced in relation to breeding status.

In several mammals, reproductive inhibition occurs due to a reluctance to mate with kin or familiar animals (Bennett et al., 1996, 1997). Certainly, we find no evidence to suggest that there are physiological mechanisms underlying reproductive inhibition or that aggression by other females is likely to act as a behavioural mechanism (Waterman, 2002; Pettitt, 2006). Alternatively, in social Cape ground squirrel colonies non-breeders may refrain from sexual activity within the group because of their genetic relatedness to males in the group. Although the majority of males disperse from their colony of birth at 10 months of age, up to $25 \%$ of males in Namibia and $62 \%$ in South Africa (Waterman, 1995; unpubl. data) delay dispersal and remain living in their natal burrow way beyond sexual maturity. These males continue to sleep and interact with their family group and can attempt to breed with related females (Waterman, 1995; unpubl. data). Thus, socially induced suppression of reproduction does not result from a decreased pituitary secretion of LH. We argue, therefore, that non-breeding females may refrain from sexual activity as a result of self-restraint. Here, individuals avoid reproduction due to potential conflict or otherwise due to incest taboos (Snowdon, 1996). Pettitt (2006) found that the presence of related males, as well as the number of breeding females in the social group, significantly affected age of maturity in the same study population. Females that delay maturity may only initiate sexual activity through exposure to unrelated partners following a reduction in the number of breeding females in a colony and the eventual dispersal of the adult natal males. The reduction in the number of breeding females in the group may either occur through the death of colony members or the fission of larger colonies with three or more breeding females into smaller groups (see Waterman, 2002).

We conclude, therefore, that Cape ground squirrels do not exhibit a physiological mechanism of reproductive suppression at the level of the pituitary - we can only speculate about the level of the hypothalamic region. We cannot exclude the possibility that the ovary of the non-breeding females is less sensitive to LH but this seems unlikely. 
At least, should physiological control at the level of the ovary occur, the mechanism is unlike several other mammals, where control is evident at the hypothalamic level. In addition, the lack of a dominance hierarchy in female groups suggests that dominant control does not underlie reproductive skew or suppression among female ground squirrels. Alternatively, delayed maturity within colonies may be governed by selfrestraint.

\section{References}

Abbott, D.H. (1984). Behavioral and physiological suppression of fertility in subordinate marmoset monkeys. Am. J. Primatol. 6, 169-186.

Armitage, K.A. \& Schwartz, O.A. (2000). Social enhancement of fitness in yellowbellied marmots. Proc. Natl. Acad. Sci. USA 97, 12149-12152.

Batzli, G.O., Getz, L.O. \& Hurley, S.S. (1977). Suppression of growth and reproduction of microtine rodents by social factors. J. Mammal. 58, 583-591.

Bennett, N.C. (1994). Reproductive suppression in social Cryptomys damarensis colonies: a lifetime of socially-induced sterility in males and females. J. Zool. (Lond.) 234, 25-39.

Bennett, N.C., Faulkes, C.G. \& Molteno, A.J. (1996). Reproductive suppression in subordinate, non-breeding female Damaraland mole-rats: two components to a lifetime of socially-induced infertility. Proc. Roy. Soc. Lond. Ser. B. - Biol. Sci. 263, 1599-1603.

Bennett, N.C., Faulkes, C.G. \& Spinks, A.C. (1997). LH responses to single doses of exogenous GnRH by social Mashona mole-rats: a continuum of socially-induced infertility in the family Bathyergidae. Proc. Roy. Soc. Lond. Ser. B. -Biol. Sci. 264, 1001-1006. 
Bennett, N.C., Jarvis, J.U.M., Faulkes, C.G. \& Millar, R.P. (1993). L. H. responses to single doses of exogenous GnRH by freshly captured Damaraland mole-rats, Cryptomys damarensis. J. Reprod. Fertil. 99, 81-86.

Blumstein, D.T. \& Armitage, K.B. (1999). Cooperative breeding in marmots. Oikos 84, 369-382.

Cant, M.A. (2000). Social control of reproduction in banded mongooses. Anim. Behav. 59, 147-158.

Chard, T. (1987). An introduction to radioimmunoassay and related techniques. 3rd edn. Amsterdam: Elsevier.

Clutton-Brock, T.H. (2002). Breeding together: kin selection and mutualism in cooperative vertebrates. Science 296, 69-72.

Clutton-Brock, T.H., MacColl, A.D.C., Chadwick, P., Gaynor, D., Kansky, R. \& Skinner, J.D. (1999). Reproduction and survival of suricates (Suricata suricatta) in the Kalahari Gemsbok Park. Afr. J. Ecol. 37, 69-80.

Coppola, D.M. \& Vandenbergh, J.G. (1985). Effects of density, duration of grouping and age of urine stimulus on the puberty delay pheromone in female mice. J. Reprod. Fertil. 73, 517-522.

Creel, S.R. \& Waser, P.M. (1991). Failures of reproductive suppression in dwarf mongooses (Helogale parvula), accident or adaptation? Behav. Ecol. Sociobiol. 2, 7-15.

Creel, S.R. \& Waser, P.M. (1997). Variation in reproductive suppression among dwarf mongooses: interplay between mechanisms and evolution. In Cooperative breeding in 
mammals: 150-170. Solomon, N.G. \& French, J.A. (Eds). Cambridge: Cambridge University Press.

van Damme, M.P., Robertson, D.M. \& Diczfalusy, E. (1974). An improved in vitro bioassay method for measuring luteinising hormone (LH) activity using mouse Leydig cell preparations. Acta Endocrinol. 77, 655-671.

Emlen, S.T. (1996). Reproductive sharing in different types of kin associations. Am. Nat. 148, 756-763.

Emlen, S.T. (1997). Predicting family dynamics in social vertebrates. In Behavioural ecology: an evolutionary approach: 228-253. Krebs, J.R. \& Davies, N.B. (Eds). Oxford: Blackwell Science.

Faulkes, C.G., Abbott, D.H. \& Jarvis, J.U.M. (1991). Social suppression of reproduction in male naked mole-rats, Heterocephalus glaber. J. Reprod. Fertil. 91, 593-604.

Faulkes, C.G., Abbott, D.H., Jarvis, J.U.M. \& Sherriff, F.E. (1990). LH responses of female naked mole-rats, Heterocephalus glaber, to single and multiple doses of exogenous GnRH. J. Reprod. Fertil. 89, 317-323.

Faulkes, C.G. \& Bennett, N.C. (2001). Family values: group dynamics and social control of reproduction in African mole-rats. Trends Ecol. Evol. 16, 184-190.

Harlow, C.R., Gems, S., Hodges, J.K. \& Hearn, J.P. (1984). The relationship between plasma progesterone and the timing of ovulation and early embryonic development in the marmoset monkey (Callithrix jacchus). J. Zool. (Lond.) 201, 272-282.

Herzig-Straschil, B. (1978). On the biology of Xerus inauris (Zimmermann, 1780) (Rodentia, Sciuridae). Z. Säugtierkd. 43, 262-278. 
Hodges, J.K., Cottingham, P., Summers, P.M. \& Yingnan, L. (1987). Controlled ovulation in the marmoset monkey (Callithrix jacchus), with human chorionic gonadotrophin following prostaglandin induced luteal regression. Fertil Steril. 48, 299305.

Koprowski, J.L. (2002). Handling tree squirrels with a safe and efficient restraint. Wildl. Soc. B. 30, 101-103.

Kruczek, M. \& Marchlewska-Koj, A. (1986). Puberty delay of bank vole females in a high-density population. Biol. Reprod. 35, 537-541.

-

Massey, A. \& Vandenbergh, J.G. (1980). Puberty delay by a urinary cue from female house mice in feral populations. Science 209, 821-822.

-

Millar, R.P., Flanagan, C.A., de Milton, R.C. \& King, J.A. (1989). Chimeric analogues of vertebrate gonadotrophin releasing hormones comprising substitutions of the variant amino acids in positions 5, 7 and 8. J. Biol. Chem. 264, 21007-21013.

O'Riain, M.J., Bennett, N.C., Brotherton, P.N.M., Mcilrath, G. \& Clutton-Brock, T.H. (2000). Reproductive suppression versus inbreeding avoidance in wild populations of cooperatively breeding suricates. Behav. Ecol. Sociobiol. 48, 471-477.

Pettitt, B.A. (2006). Reproductive delay in female Cape ground squirrels (Xerus inauris). MSc thesis, University of Central Florida, Orlando.

Schwartz, O.A. \& Armitage, K.B. (2005). Weather influences on demography of the yellow-bellied marmot (Marmota flaviventris). J. Zool. (Lond.) 265, 73-79.

Snowdon, C.T. (1996). Infant care in cooperatively breeding species. Adv. Stud. Behav. 25, 643-689. 
Vehrencamp, S.L. (1983). A model for the evolution of despotic versus egalitarian societies. Anim. Behav. 31, 667-682.

Wasser, S.K. \& Barash, D.P. (1983). Reproductive suppression amongst female mammals: implications for biomedicine and sexual selection theory. Q. Rev. Biol. 58, 513-538.

Waterman, J.M. (1995). The social organisation of the Cape ground squirrel (Xerus inauris; Rodentia: sciuridae). Ethology 101, 130-147.

Waterman, J.M. (1996). Reproductive biology of a tropical non-hibernating ground squirrel. J. Mammal. 77, 134-146.

Waterman, J.M. (1998). Mating tactics of male Cape ground squirrels, Xerus inauris: consequences of year-round breeding. Anim. Behav. 56, 459-466.

Waterman, J.M. (2002). Delayed maturity, group fission and the limits of group size in female Cape ground squirrels (Sciuridae: Xerus inauris). J. Zool. (Lond.) 256, 113-120.

Wingfield, J.C. \& Sapolsky, R.M. (2003). Reproduction and resistance to stress: when and how. J. Neuroendocrinol. 15, 711-724. 


\section{Figures and tables}

Figure 1 Concentrations of plasma bioactive luteinizing hormone (LH) (means $\pm \mathrm{SE}$ ) in breeding female Cape ground squirrels Xerus inauris, pre- $(0 \mathrm{~min})$ and post- $(20 \mathrm{~min}$ after) a single subcutaneous injection of GnRH or a saline placebo. LH increased significantly after injection of $\mathrm{GnRH}$, but not the saline placebo.

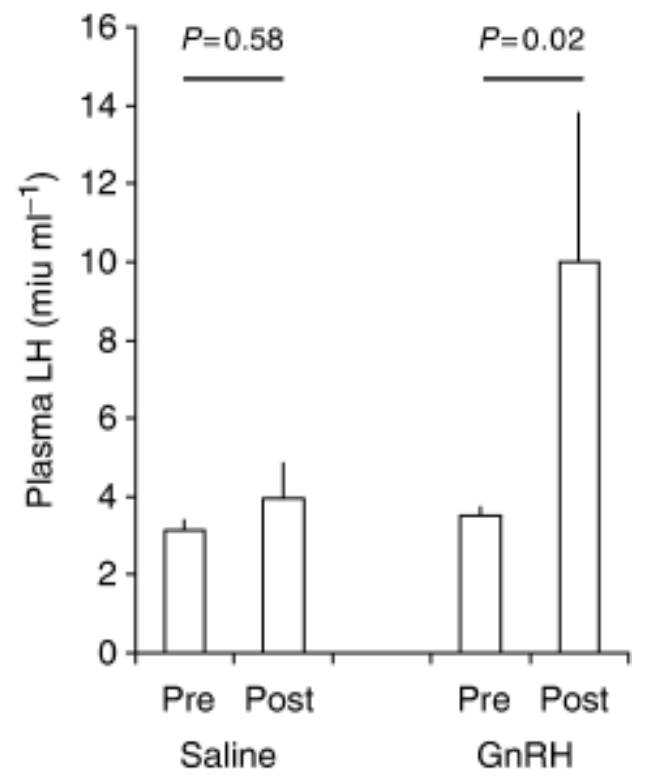


Figure 2 Effect of breeding status (breeding, non-breeding) of female Cape ground squirrels Xerus inauris on concentrations of plasma bioactive luteinizing hormone (LH) (means \pm SE), pre- $(0 \mathrm{~min})$ and post- ( $20 \mathrm{~min}$ after $)$ a single subcutaneous injection of $\mathrm{GnRH}$. No effect of status was recorded, although LH increased significantly following the GnRH challenge. Sample sizes are indicated above the histogram.

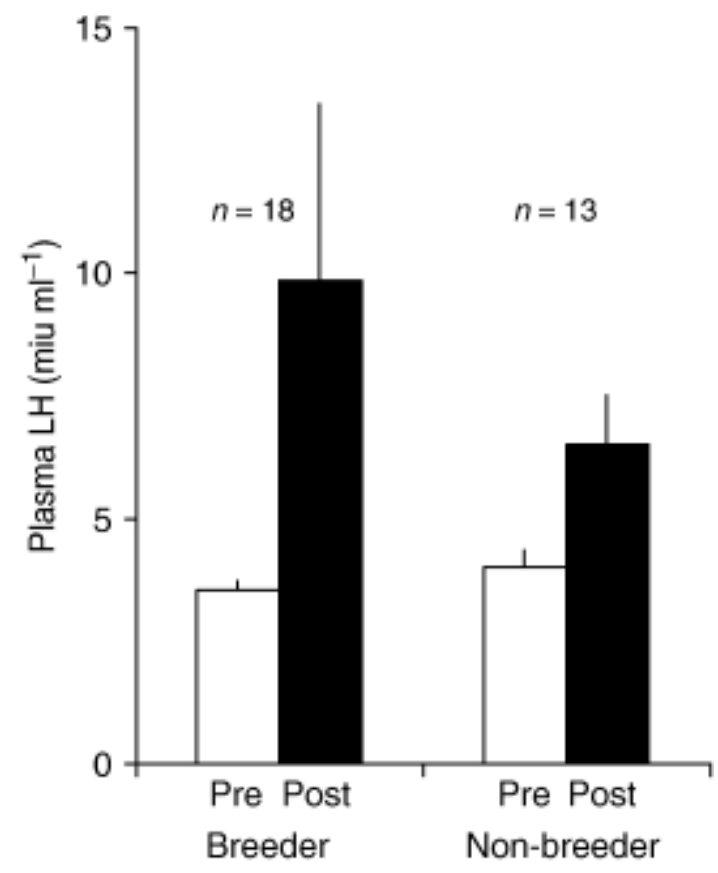


Figure 3 Effect of season (summer, winter) on concentrations of plasma bioactive luteinizing hormone (LH) (means $\pm \mathrm{SE}$ ) for female Cape ground squirrels Xerus inauris pre- $(0 \mathrm{~min})$ and post- (20 min after) a single subcutaneous injection of GnRH.

Circulating levels of LH were higher during the winter, while LH increased significantly in both seasons following the GnRH challenge. Sample sizes are indicated above the histogram.

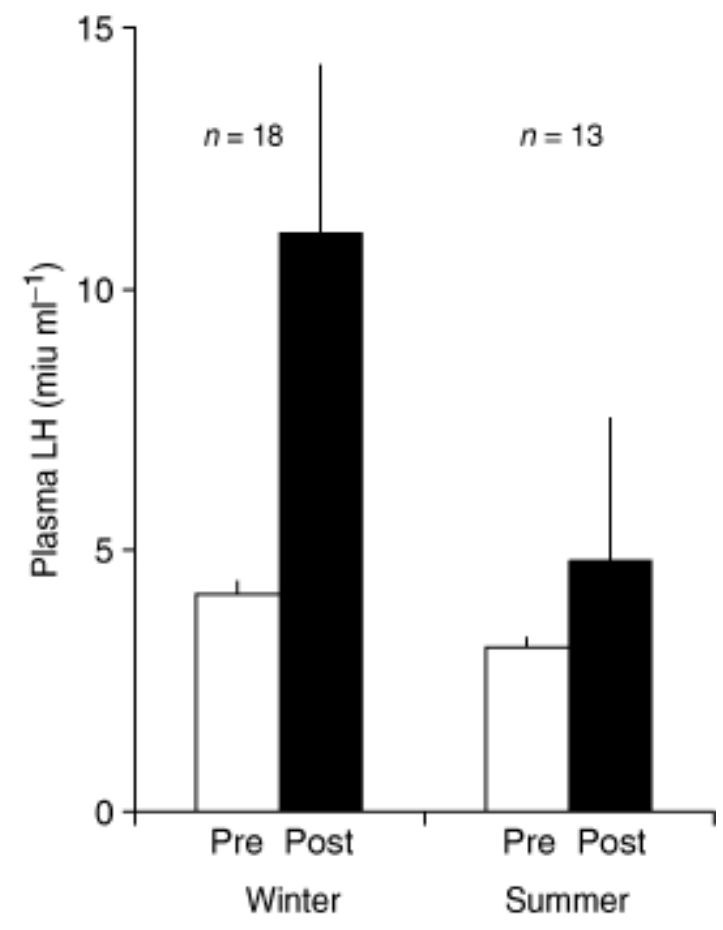

